\title{
IPF: time for the (ciliary) beat generation?
}

\author{
Sarah Wiscombe, ${ }^{1}$ Ian A Forrest, ${ }^{2}$ A John Simpson ${ }^{1,2}$
}

Idiopathic pulmonary fibrosis (IPF), the most common of the idiopathic interstitial pneumonias remains a disabling, progressive lung disease with extremely poor prognosis, in which no pharmacological intervention significantly alters outcome. ${ }^{1}$ A relatively poor understanding of the complex pathophysiology of IPF continues to hinder the identification of effective therapies, and of patients most likely to benefit from existing treatments.

Usual interstitial pneumonia is the histological hallmark of IPF, characterised by temporospatial heterogeneity in which normal lung is interspersed with areas of subpleural interstitial fibrosis, loss of normal alveolar architecture and the presence of fibroblastic foci. ${ }^{2}$ Histological sections may also show 'bronchiolisation' of the distal airway with alveolar structures replaced by enlarged airspaces (forming characteristic honeycomb cysts) lined by epithelial cells more akin to proximal airway epithelium, often ciliated and mucus producing. ${ }^{3}$ The potential importance of mucus production in the pathogenesis of IPF has been highlighted by a landmark study demonstrating that a common variant within the putative promoter region of MUC5B (an airway mucin gene) is strongly associated with familial interstitial pneumonia and IPF. ${ }^{5}$ Moreover, subjects with IPF had significantly higher expression of MUC5B protein levels compared with controls, and this was localised to areas of lung fibrosis. It has been hypothesised that excessive MUC5B may impair the host response to alveolar injury through excess mucus plugging and impaired clearance of inhaled substances and micro-organisms. Alternative theories propose that MUC5B may interfere directly with alveolar repair mechanisms, either through disruption of interactions between type II alveolar epithelial cells and extracellular matrix, or by interfering with properties of surfactant and promoting alveolar collapse.

\footnotetext{
${ }^{1}$ Institute of Cellular Medicine, Newcastle University, Newcastle upon Tyne, UK; ${ }^{2}$ Newcastle Interstitial Lung Disease Clinic, Royal Victoria Infirmary, Newcastle upon Tyne, UK

Correspondence to Professor John Simpson, Institute of Cellular Medicine, Medical School, Newcastle

University, 4th Floor, William Leech Building, Newcastle upon Tyne NE2 4HH, UK; j.simpson@ncl.ac.uk
}

While there has been a proliferation in interest surrounding mucin production in IPF, the importance of cilia has received much less attention. The fascinating gene expression study from Yang et $a l^{6}$ in this edition of Thorax seems set to change this. The authors performed comparative RNA microarray analysis on lung tissue from 119 patients with well-characterised IPF and from patients with brain death whose lungs were considered unusable for lung transplantation. Genes differentially expressed in IPF were interrogated using hierarchical clustering, yielding two subgroups of IPF differentiated by distinct expression profiles. Intriguingly, the strongest signal distinguishing the two cohorts of patients with IPF appeared to come from cilia-associated genes and their structural components (DNAH6, DNAH7, DNAI1 and RPGRIP1L) as well as MUC5B. The authors broadly replicated their findings in lung tissue from an independent cohort with IPF. Interestingly, the two IPF cohorts were indistinguishable clinically, but the 'cilia-high' group had more histological honeycombing in tissue taken from the area immediately adjacent to that used for microarray analysis.

This meticulous study adds important new knowledge in IPF. Consistent findings in two independent cohorts of wellcharacterised patients, relevant tissue, strong genetics expertise and decent sample sizes represent obvious strengths of the study, and increase the likelihood that the observation is 'real'. Furthermore, the biological plausibility of the findings is enticing, because ciliated 'bronchiolised' epithelium is seen in honeycomb cysts. ${ }^{7}$ While the motile functions of cilia are well described, there has been a recent explosion in understanding of the role of the primary cilium in 'sensing' the cellular microenvironment, and in cell motility. Interestingly, ciliary chemosensing appears to be mediated via molecules already implicated in the pathogenesis of IPF such as Sonic-Hedgehog, plateletderived growth factor receptor and canonical Wnt. ${ }^{8}$ Furthermore, primary cilia coordinate a series of signalling pathways critical to fibroblast migration in response to wound healing. In particular platelet-derived growth factor receptor $\alpha$ is compartmentalised to the primary cilium to activate pathways that regulate reorganisation of the cytoskeleton. ${ }^{9}$ Cigna et al ${ }^{10}$ recently demonstrated that hedgehog pathways were reactivated in the IPF lung and have significant crosstalk with transforming growth factor $\beta$ signalling in lung fibroblasts. Wnt and $\beta$-catenin are located on the membrane and basal body of the primary ciliumcanonical Wnt signalling inactivates glycogen synthase kinase $3 \beta$, thus stabilising $\beta$-catenin, which enters the nucleus to activate Wnt target genes (including fibronectin, matrix metalloproteinase 7 , cyclins, axins and vascular endothelial growth factor). It has been suggested that upregulation of Wnt signalling may inhibit the ability of type II alveolar epithelial cells to differentiate into type I alveolar epithelial cells in response to injury (thus disrupting repair mechanisms). ${ }^{11}$

As with all clinical IPF studies (however meticulous), some aspects of the paper by Yang et al must be interpreted cautiously. The cohort with upregulated cilia-associated genes had far more histological honeycomb cysts, and the implication may simply be that more 'bronchiolised', and therefore ciliated, tissue was available to sample (though the authors provide a fair argument against this bias playing a significant part). Also, the patients with IPF all had lung biopsies, and were younger than the average patient with IPF. In addition, the majority of the control group is likely to have suffered significant trauma, all will have been mechanically ventilated, and the implication is that there was sufficient inflammatory change to preclude use of these lungs for transplantation-these factors may have skewed the panel of genes selected to distinguish IPF cohorts. Finally, the specific cell type(s) producing the upregulation in ciliary genes remains unknown.

Equally, as with all fresh, important clinical research, the study stimulates more questions than it answers. One key question that remains to be answered is whether the two IPF cohorts differed in terms of outcomes such as mortality, loss of lung function or response to treatment? Also, could the study findings simply reflect duration of disease? Certainly one cohort of IPF had surprisingly little microscopic honeycombing-perhaps this could reflect sampling bias, or perhaps the natural history of the disease would be for these patients to go on and develop histological microcysts? Are the transcriptional changes actually associated with changes in cellular function? Perhaps most importantly, is upregulation of cilia-associated genes an essential component of disease pathogenesis, a compensatory and appropriate defence mechanism, or a functionally unimportant reaction to more important pathogenetic processes? 
Despite these considerations, the potential importance of this study is considerable. Certainly it highlights the ability of good genetic studies to generate new hypotheses and new avenues for exploration. In this particular case it throws attention onto the bronchiolisation process. Considerable focus has centred on epithelial-tomesenchymal transition in recent years, but perhaps we have ignored the potential of stressed alveolar epithelium to transform into an entirely different epithelial configuration(s). The tantalising possibility, of course, is that secretion of mucin and upregulation of cilia-associated genes may be intrinsically linked. The authors of the present study are ideally placed to begin to address this issue, and their recent finding that specific polymorphisms in the MUC5B promoter region may be associated with better prognosis in $\mathrm{IPF}^{12}$ highlights the complex interplay between biological factors and pathogenesis.

Of course, we remain frustratingly distant from the day when we can perform something like a transbronchial biopsy and have its transcriptome reliably inform us how best to manage patients with IPF. Nevertheless, a real momentum seems to be building in terms of high quality clinical studies identifying potentially important processes in the biology of IPF. The important next step is obviously to determine whether these can be manipulated to therapeutic advantage. In this regard, we currently await results from the final part of the PANTHER-IPF trial, ${ }^{13}$ assessing whether the mucolytic, antioxidant $\mathrm{N}$-acetylcysteine has beneficial effects in IPF. Meantime, the elegant study from Yang et al suggests that perhaps the ciliary beat is the beat to follow. Perhaps IPF researchers are ready to answer the question Jack Kerouac himself posed in On The Road-'You boys going to get somewhere, or just going?'

Contributors The authors wrote and approved the manuscript.

Competing interests None.

Provenance and peer review Commissioned; externally peer reviewed.

To cite Wiscombe $S$, Forrest IA, Simpson AJ. Thorax 2013;68:1088-1089.

Published Online First 4 September 2013

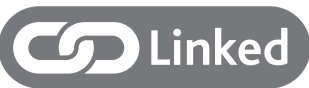

http://dx.doi.org/10.1136/thoraxjnl-2012-202943

Thorax 2013;68:1088-1089.

doi:10.1136/thoraxjnl-2013-203928

\section{REFERENCES}

1 Raghu G, Collard HR, Egan JJ, et al. An official ATS/ ERS/JRS/ALAT statement: idiopathic pulmonary fibrosis: evidence-based guidelines for diagnosis and management. Am J Respir Crit Care Med 2011:183:788-824.

2 Khalil N, Xu YD, O'Connor R, et al. Proliferation of pulmonary interstitial fibroblasts is mediated by transforming growth factor-beta-1-induced release of extracellular fibroblast growth-factor-2 phosphorylation of p38 MAPK and JNK. J Biol Chem 2005;280:43000-9.

3 Katzenstein ALA, Myers JL. Idiopathic pulmonary fibrosis: clinical relevance of pathologic classification. Am J Respir Crit Care Med 1998;157: 1301-15.

4 Hoo ZH, Whyte MKB. Idiopathic pulmonary fibrosis. Thorax 2012;67:742-6.

5 Seibold MA, Wise AL, Speer MC, et al. A common MUC5B promoter polymorphism and pulmonary fibrosis. N Engl J Med 2011;364:1503-12.

6 Yang IV, Coldren CD, Leach SM, et al. Expression of cilium-associated genes defines novel molecular subtypes of idiopathic pulmonary fibrosis. Thorax 2013:68:1114-21.

7 Seibold MA, Smith RW, Urbanek C, et al. The idiopathic pulmonary fibrosis honeycomb cyst contains a mucociliary pseudostratified epithelium. PLOS ONE 2013;8:e58658.

8 Fliegauf $\mathrm{M}$, Benzing $\mathrm{T}$, Omran $\mathrm{H}$. When cilia go bad: cilia defects and ciliopathies. Nat Rev Mol Cell Biol 2007:8:880-93.

9 Christensen ST, Veland IR, Schwab A, et al. Analysis of primary cilia in directional cell migration in fibroblasts. Methods Enzymol 2013;525: 45-58.

10 Cigna N, Moshai EF, Brayer S, et al. The Hedgehog system machinery controls transforming growth factor- $\beta$-dependent myofibroblastic differentiation in humans. Am J Pathol 2012;181:2126-37.

11 Henderson WR Jr, Chi EY, Ye X, et al. Inhibition of Wnt/beta-catenin/CREB binding protein (CBP) signalling reverses pulmonary fibrosis. Proc Nat/ Acad Sci USA 2010;345:517-25.

12 Pelito AL, Zhang Y, Fingerlin TE, et al. Association between the MUC5B promoter polymorphism and survival in patients with idiopathic pulmonary fibrosis. JAMA 2013;309:2232-9.

13 Raghu G, Anstrom KJ, King TE Jr, et al.; Idiopathic Pulmonary Fibrosis Clinical Research Network. Prednisone, azathioprine, and $\mathrm{N}$-acetylcysteine for pulmonary fibrosis. N Engl J Med 2012;366:1968-77. 